\title{
DPROTEIN FOLDING
}

\section{Handling the stress}

DOI:

10.1038/nrm1955
BAX and BAK are mitochondrial proapoptotic proteins that function in intrinsic cell-death pathways. However, a report in Science now shows that BAX and BAK also function at the endoplasmic reticulum (ER) membrane, and reveals a new role for these members of the core apoptotic machinery in the stressassociated unfolded-protein response (UPR).

In higher eukaryotes, ER stress stimulates three distinct UPR-signalling pathways through sensors that include IRE1 $\alpha$ (inositol-requiring transmembrane kinase and endonuclease-1 $\alpha$ ), PERK (protein kinaselike ER kinase) and ATF6 (activation of transcription factor-6). Cells from mice that are deficient in both Bax and $B a k$ are resistant to proapoptotic agents that induce the UPR, which

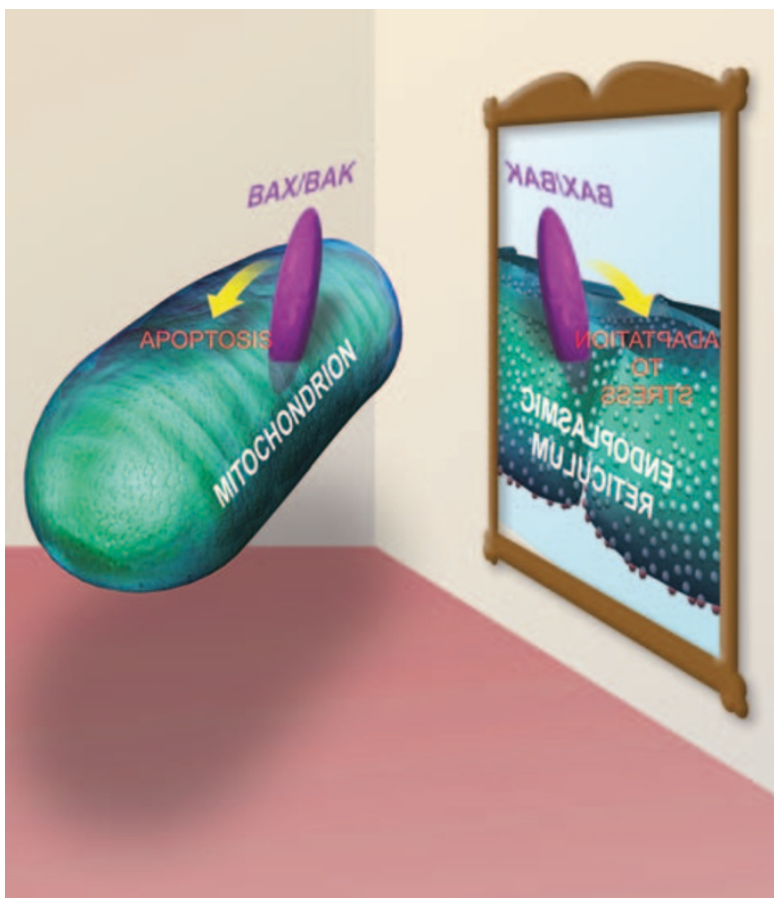

Image courtesy of E. Smith, Dana Farber Cancer Institute, Boston, USA. prompted the authors to further investigate the connection between these proapoptotic proteins and UPR signalling events in vivo.

Hetz et al. generated conditional Bax Bak double-knockout mice and used an in vivo model for ER stress, which involved the intraperitoneal injection of tunicamycin into the liver and kidney. Bax Bak double-knockout mice responded abnormally to tunicamycin-induced ER stress in the liver; they displayed extensive tissue damage and decreased expression of the IRE1 $\alpha$-substrate X-box-binding protein-1 (XBP1) and its target genes. Hetz et al. next showed that this phenotype was associated with cellular dysfunction rather than cell death. This finding led the authors to postulate that BAX and BAK might have an important role in adaptation responses to ER stress in vivo through the modulation of IRE1 $\alpha$ signalling.

How can these mitochondrial proteins modulate an ER response?
The authors analysed different signalling pathways in Bax Bak doubleknockout cells and first showed that these cells display a phenotype that is similar to that of IRE $1 \alpha$-deficient cells after ER-stress induction. Then, using organelle-specific reconstitution assays and ER-targeted versions of BAK, Hetz et al. not only found that BAK expression at the ER membrane is required for IRE1 $\alpha$ signalling, but also showed that both BAK and BAX directly interact with the cytosolic domain of IRE1 $\alpha$, probably stabilizing its active form.

Whether these proteins function as stress sentinels that connect stress signals to the proapoptotic machinery - under circumstances when cellular homeostasis is irreversibly altered - remains to be seen.

\section{Ekat Kritikou}

ORIGINAL RESEARCH PAPER Hetz, C. et al. Proapoptotic BAX and BAK modulate the unfolded protein response by a direct interaction with IRE1 $\alpha$. Science 312, 572-576 (2006)
Whether

these proteins

function

as stress

sentinels ...

remains to be

seen.

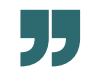

